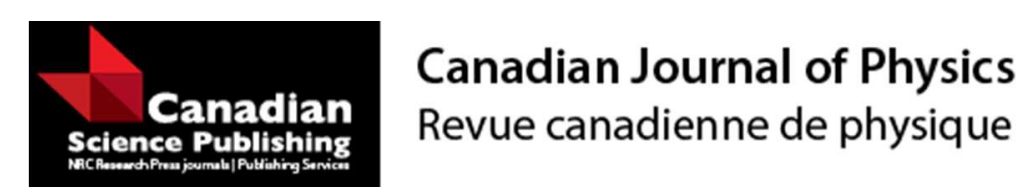

\title{
Oscillator strengths for spin-changing P-D transitions in He $I$ including the effect of a finite nuclear mass and intermediate coupling
}

\begin{tabular}{|r|l|}
\hline Journal: & Canadian Journal of Physics \\
\hline Manuscript ID & cjp-2016-0890 \\
\hline Manuscript Type: & Article \\
\hline Date Submitted by the Author: & $30-$ Nov-2016 \\
\hline Complete List of Authors: & $\begin{array}{l}\text { Morton, Donald C.; National Reseach Council, Herzberg Astronomy and } \\
\text { Astrophysics } \\
\text { Drake, Gordon; University of Windsor, }\end{array}$ \\
\hline Keyword: & $\begin{array}{l}\text { helium, atomic spectra, transition probabilities, nuclear mass, relativistic } \\
\text { effects }\end{array}$ \\
\hline
\end{tabular}

\section{SCHOLARONE ${ }^{\text {Tw }}$ \\ Manuscripts}




\title{
Oscillator strengths for spin-changing $\mathbf{P}-\mathrm{D}$ transitions in He I including the effect of a finite nuclear mass and intermediate coupling
}

\author{
Donald C. Morton ${ }^{1}$ and G. W. F. Drake ${ }^{2}$ \\ 1 Herzberg Astronomy and Astrophysics, National Research Council of Canada, \\ 1985 Rue de la Régence, St.-Bruno-de-Montarville, QC, J3V 4B7, Canada \\ 2 Department of Physics, University of Windsor, Windsor, ON, N9B 3P4, \\ Canada \\ E-mail: don.morton@nrc.gc.ca, gdrake@uwindsor.ca
}

\begin{abstract}
We have calculated the electric dipole (E1) oscillator strengths and spontaneous decay rates for 12 spin-changing $\mathrm{P}-\mathrm{D}$ transitions of atomic helium. We included the effects of the finite nuclear mass and the anomalous magnetic moment of the electron. The specific transitions for ${ }^{4} \mathrm{He}$ are $n{ }^{1} \mathrm{P}_{1}^{\mathrm{o}}-n^{\prime}{ }^{3} \mathrm{D}_{1,2}$ and $n{ }^{3} \mathrm{P}_{1,2}^{\mathrm{o}}-n^{\prime}{ }^{1} \mathrm{D}_{2}$ with $n=2,3,4$ and $n^{\prime}=3,4$. To include the effects of intermediate coupling between pure $L S$ and $j j$, we used the Breit formulation for the spin-orbit and spin-other-orbit operators and combined the results of the exact diagonalization of the $2 \times 2$ energy matrix with pseudostate expansions to perform the perturbation sums over intermediate states. We calculated both the length and velocity gauges as a check on numerical accuracy and the validity of the transition operators.
\end{abstract}

\section{Introduction}

With this paper we wish to extend our previous calculations of spin-changing radiative transitions in neutral helium to $\mathrm{P}$ - D transitions and include the effects of the finite nuclear mass and the anomalous magnetic moment of the electron. In Paper I [1] we published oscillator strengths ( $f$-values) and transition rates ( $A$-values) for the lowest $\mathrm{S}-\mathrm{P}$ and $\mathrm{P}-\mathrm{D}$ transitions and in Paper II [2] the five lowest $\mathrm{S}-\mathrm{P}$ transitions. These calculations summed the spin-dependent Breit interaction as a first-order perturbation over all intermediate states, but assumed infinite nuclear mass. In Paper III [3] we applied the same perturbation analysis to $24 \mathrm{~S}-\mathrm{P}$ transitions and included the electron anomaly and the true nuclear mass, which partially cancel each other so it is important to include both. The present paper extends those results to $12 \mathrm{P}-\mathrm{D}$ transitions with corrections to the wave functions and operators for the nuclear mass and electron anomaly and the operators for the electron anomaly. In a recent related paper [4] we applied the same theoretical approach to the helium-like ions of C V, N VI and O VII.

In another earlier paper [5] we tabulated an extensive set of He I $f$-values for both spin-permitted and spin-forbidden transitions $n^{2 S+1} L_{J}-n^{\prime 2 S^{\prime}+1} L_{J}^{\prime}$ with $n, n^{\prime} \leq 10 ; S, S^{\prime}=0,1 ; L, L^{\prime} \leq 6$, all for infinite nuclear mass. The relevant physics is different for high $L$ because the electrostatic singlet-triplet splitting becomes small relative to the spin-dependent Breit interaction, and so only $\mathbf{L}+\mathbf{S}=\mathbf{J}$ remains a 
good quantum number. As $L$ increases, there is a progressive change from pure $L S$ coupling to pure $j j$-coupling for high $L$ [6]. In the intermediate case, the dominant mixing is between the pair of $L S$-coupled states with the same $n, L$, and $J$, but different $S$, for example the singlet-triplet pair $1 s 3 d^{1} \mathrm{D}_{2}$ and $1 s 3 d^{3} \mathrm{D}_{2}$. Consequently Ref. [5] included only this mixing by diagonalizing the corresponding $2 \times 2$ matrix in place of a first-order perturbation sum over all intermediate states, which fails where the mixing is strong. This use of exact diagonalization is necessary for transitions such as $3{ }^{1} \mathrm{D}_{2}-4{ }^{1} \mathrm{~F}_{3}$ and is almost adequate for $n{ }^{1} \mathrm{~S}_{0}-n{ }^{3} \mathrm{P}_{1}$ and $n{ }^{3} \mathrm{~S}_{1}-n{ }^{1} \mathrm{P}_{1}$, where the principal quantum numbers are the same, but inadequate for transitions such as $1{ }^{1} \mathrm{~S}_{0}-2{ }^{3} \mathrm{P}_{1}$ or $2{ }^{3} \mathrm{~S}_{1}-3{ }^{1} \mathrm{P}_{1}$. For the $\mathrm{P}-\mathrm{D}$ transitions in this paper we use a combination of exact diagonalization and summation over intermediate states to treat the case of intermediate coupling between $L S$ and $j j$.

In Sections 2 and 3 below we repeat the theoretical discussion in Paper III [3] for the true nuclear mass and the electron anomaly and show how the summation over intermediate states is modified to use the exact diagonalization of the ${ }^{1} \mathrm{D}_{2}$ and ${ }^{3} \mathrm{D}_{2}$ states. We use reduced atomic units in which we relate lengths $r$ and energies $\epsilon$ to laboratory values by $R=a_{0}\left(m_{\mathrm{e}} / \mu\right) r$ and $E=\alpha^{2} m_{\mathrm{e}} c^{2}\left(\mu / m_{\mathrm{e}}\right) \epsilon$. Here $a_{0}, \alpha, m_{\mathrm{e}}$ and $c$ are the familiar atomic constants [7], $\mu=m_{\mathrm{e}} M /\left(m_{\mathrm{e}}+M\right)$ is the reduced mass of the electron, and $M$ is the mass of the nucleus. The usual atomic units (a.u.) result if $M=\infty$.

\section{Theory for sums over intermediate states}

Solution of the mass-polarized nonrelativistic Schrödinger equation [8]

$$
\begin{aligned}
& H_{\mathrm{M}} \psi_{M}^{0}=\left[-\frac{1}{2}\left(\nabla_{1}^{2}+\nabla_{2}^{2}\right)-\frac{\mu}{M}\left(\nabla_{1} \cdot \nabla_{2}\right)\right. \\
& \left.-Z\left(\frac{1}{r_{1}}+\frac{1}{r_{2}}\right)+\frac{1}{r_{12}}\right] \psi_{M}^{0} \\
& \quad=\varepsilon_{M}^{0} \psi_{M}^{0}
\end{aligned}
$$

provides the unperturbed energies $\epsilon_{M}^{0}$ and wave functions $\psi_{M}^{0}$ [9] for nuclear mass $M$. This equation is in reduced-mass a.u., so we must multiply these energies by $\mu / m_{\mathrm{e}}$ to obtain a.u. and multiply all operators used with these $\psi_{M}^{0}$ by one power of $\mu / m_{\mathrm{e}}$ for each power of $1 / r$ in the operator. These wave functions contain no spin corrections.

When the electron spin changes, the relevant spin-orbit (SO) and spin-other-orbit (SOO) perturbers are the respective Breit operators

$$
\begin{aligned}
B_{3 Z}= & \frac{\alpha^{2} Z}{4}\left[\left(\frac{\mathbf{r}_{1}}{r_{1}^{3}} \times \mathbf{p}_{1}\right) \cdot \sigma_{1}+\left(\frac{\mathbf{r}_{2}}{r_{2}^{3}} \times \mathbf{p}_{2}\right) \cdot \sigma_{2}\right] \\
B_{3 e}= & \frac{\alpha^{2}}{4 r_{12}^{3}}\left[\left(\mathbf{r}_{12} \times \mathbf{p}_{2}-\mathbf{r}_{12} \times \mathbf{p}_{1}\right) \cdot\left(\sigma_{1}+\sigma_{2}\right)\right. \\
& \left.+\left(\mathbf{r}_{12} \times \mathbf{p}_{2}\right) \cdot \sigma_{1}-\left(\mathbf{r}_{12} \times \mathbf{p}_{1}\right) \cdot \sigma_{2}\right] .
\end{aligned}
$$

In [10] Stone formulated the finite-mass corrections and included the effects of the anomalous magnetic moment of the electron $a_{\mathrm{e}} \simeq \alpha /(2 \pi)$. Following Drake and Yan [11], who approximated Stone's $m_{\mathrm{e}} / M$ with $y=\mu / M$, the SO and SOO operators become

$$
B_{3 \mathrm{ZMa}_{\mathrm{e}}}=\left(\mu / m_{\mathrm{e}}\right)^{3}\left[\left(1+2 a_{\mathrm{e}}+2 y+2 a_{\mathrm{e}} y\right) B_{3 Z}\right.
$$




$$
\begin{gathered}
\left.+\left(1+a_{\mathrm{e}}\right) y \Delta_{3}\right] \\
B_{3 \mathrm{eMa}_{\mathrm{e}}}=\left(\mu / m_{\mathrm{e}}\right)^{3}\left[B_{3 \mathrm{e}}+\frac{a_{\mathrm{e}} \alpha^{2}}{4 r_{12}^{3}}\left(\mathbf{r}_{12} \times \mathbf{p}_{2}-\mathbf{r}_{12} \times \mathbf{p}_{1}\right)\right. \\
\left.\cdot\left(\sigma_{1}+\sigma_{2}\right)\right] \\
\Delta_{3}=\frac{\alpha^{2} Z}{2}\left[\left(\frac{\mathbf{r}_{1}}{r_{1}^{3}} \times \mathbf{p}_{2}\right) \cdot \sigma_{1}+\left(\frac{\mathbf{r}_{2}}{r_{2}^{3}} \times \mathbf{p}_{1}\right) \cdot \sigma_{2}\right]=2 B_{3 Z} .
\end{gathered}
$$

where the $a_{\mathrm{e}}$ term in (5) contributes only for triplet - triplet transitions. Note that the equations corresponding to (4) and (5) in Ref. [11] are in reduced mass atomic units, so the prefactors there are $(\mu / M)^{2} \simeq 1-2 y$ instead of $(\mu / M)^{3} \simeq 1-3 y$.

The mass-corrected dipole length and velocity interaction operators are

$$
\begin{aligned}
& H_{\mathrm{I}, \mathrm{L}}=\left(\mu / m_{\mathrm{e}}\right)^{-1} Z_{r}\left(z_{1}+z_{2}\right) \\
& H_{\mathrm{I}, \mathrm{V}}=\left(\mu / m_{\mathrm{e}}\right)^{1} Z_{p}\left(\frac{\partial}{\partial z_{1}}+\frac{\partial}{\partial z_{2}}\right),
\end{aligned}
$$

where

$$
Z_{r}=\frac{M+Z m_{\mathrm{e}}}{M+N m_{\mathrm{e}}}, \quad Z_{p}=\frac{M+Z m_{\mathrm{e}}}{M}
$$

and the number of electrons $N=2$. As outlined in the appendix of [5], these last factors correct the dipole operators for the motion of the nucleus relative to the centerof-mass. When $N \neq Z$, there are additional small corrections proportional to $N-Z$ in the length and velocity operators due to the motion of the centre of mass relative to the inertial frame defined by the electromagnetic field. In the absence of external fields, the centre of mass $R_{c}$ is an ignorable coordinate, so these extra terms do not contribute for spontaneous emission.

The commutator relation

$$
\begin{aligned}
& \mathrm{i}\left[H_{\mathrm{I}, \mathrm{L}}, H_{0}+B_{3 \mathrm{Z}}+B_{3 \mathrm{e}}\right] \\
& =\left(H_{\mathrm{I}, \mathrm{V}}+B_{\mathrm{I}, 3 \mathrm{Z}}+B_{\mathrm{I}, 3 \mathrm{e}}\right)
\end{aligned}
$$

relates the dipole operators $H_{\mathrm{IL}}$ and $H_{\mathrm{IV}}$ in (7) and (10), the unperturbed Hamiltonian $H_{0}$ in (1) for infinite nuclear mass and the Breit operators $B_{3 \mathrm{Z}}$ and $B_{3 \mathrm{e}}$ from (2) and (3) to the corresponding interaction operators

$$
\begin{aligned}
& B_{\mathrm{I}, 3 \mathrm{Z}}=\frac{-\alpha^{2} Z}{4}\left[\left(\frac{\mathbf{r}_{1}}{r_{1}^{3}} \times \hat{\mathbf{e}}\right) \cdot \sigma_{1}+\left(\frac{\mathbf{r}_{2}}{r_{2}^{3}} \times \hat{\mathbf{e}}\right) \cdot \sigma_{2}\right] \\
& B_{\mathrm{I}, 3 \mathrm{e}}=\frac{-\alpha^{2}}{4 r_{12}^{3}}\left[\left(\mathbf{r}_{12} \times \hat{\mathbf{e}}\right) \cdot \sigma_{1}-\left(\mathbf{r}_{12} \times \hat{\mathbf{e}}\right) \cdot \sigma_{2}\right] .
\end{aligned}
$$

Following Drake [12] these terms provide a correction to the velocity element through use of the expanded energies $\epsilon=\epsilon^{0}+\epsilon^{1}$ and wave functions $\psi=\psi^{0}+\psi^{1}$ and retaining only first-order terms so that

$$
\begin{aligned}
& \mathrm{i}\left(\epsilon_{\mathrm{a}}^{0}-\epsilon_{\mathrm{b}}^{0}\right)\left[\left\langle{ }^{3} \psi_{\mathrm{b}}^{0}\left|\mathrm{H}_{\mathrm{I}, \mathrm{L}}\right|{ }^{1} \psi_{\mathrm{a}}^{1}\right\rangle+\left\langle{ }^{3} \psi_{\mathrm{b}}{ }^{1}\left|\mathrm{H}_{\mathrm{I}, \mathrm{L}}\right|{ }^{1} \psi_{\mathrm{a}}^{0}\right\rangle\right] \\
& =\left\langle{ }^{3} \psi_{b}^{0}\left|H_{\mathrm{I}, \mathrm{V}}\right|{ }^{1} \psi_{a}^{1}\right\rangle+\left\langle{ }^{3} \psi_{b}^{1}\left|H_{\mathrm{I}, \mathrm{V}}\right|^{1} \psi_{a}^{0}\right\rangle+C_{\infty} .
\end{aligned}
$$

Thus the length matrix element multiplied by the nonrelativistic energy difference equals the velocity element plus the correction

$$
\begin{aligned}
C_{\infty} & =\frac{\alpha^{2}}{4}\left\langle{ }^{3} \psi_{b}^{0}\left|B_{\mathrm{I}, 3 \mathrm{Z}}+B_{\mathrm{I}, 3 \mathrm{e}}\right|{ }^{1} \psi_{a}^{0}\right\rangle \\
& =\frac{\sqrt{2} \alpha^{2}}{4}\left\langle{ }^{3} \psi_{b}^{0}\left|\frac{Z}{r_{1}^{3}} z_{1}-\frac{Z}{r_{2}^{3}} z_{2}+\frac{2}{r_{12}^{3}}\left(z_{1}-z_{2}\right)\right|{ }^{1} \psi_{a}^{0}\right\rangle,
\end{aligned}
$$


which depends on only the initial and final nonrelativistic states as indicated by the superscript 0 .

Enhancing (10) to include the mass and electron corrections then gives an equation connecting (4) and (5) with the corresponding extensions of (11) and (12)

$$
\begin{aligned}
& B_{\mathrm{I}, 3 Z \mathrm{ZMa}_{\mathrm{e}}}=\left(\mu / m_{\mathrm{e}}\right)^{3}\left(1+2 a_{\mathrm{e}}+2 y+2 a_{\mathrm{e}} y\right) B_{\mathrm{I}, 3 \mathrm{Z}} \\
& \Delta_{\mathrm{I}, 3 \mathrm{ZMa}_{\mathrm{e}}}=\left(\mu / m_{\mathrm{e}}\right)^{3} 2\left(y+a_{\mathrm{e}} y\right) B_{\mathrm{I}, 3 \mathrm{Z}} \\
& B_{\mathrm{I}, 3 \mathrm{eMa}}=\left(\mu / m_{\mathrm{e}}\right)^{3} B_{\mathrm{I}, 3 \mathrm{e}} .
\end{aligned}
$$

Then the correction to the sum of matrix elements with the velocity interaction operator is

$$
\begin{aligned}
C_{\mathrm{Ma}_{\mathrm{e}}}= & \left(\mu / m_{\mathrm{e}}\right)^{2} \frac{\sqrt{2} \alpha^{2}}{4} \\
& {\left[\left(1+2 a_{\mathrm{e}}+4 y+4 a_{\mathrm{e}} y\right)\left\langle{ }^{3} \mathrm{D}\left|\frac{Z}{r_{1}^{3}} z_{1}-\frac{Z}{r_{2}^{3}} z_{2}\right|{ }^{1} \mathrm{P}^{\mathrm{o}}\right\rangle\right.} \\
& \left.+\left\langle{ }^{3} \mathrm{D}\left|\frac{2}{r_{12}^{3}}\left(z_{1}-z_{2}\right)\right|{ }^{1} \mathrm{P}^{\mathrm{o}}\right\rangle\right] .
\end{aligned}
$$

where we have written the equation explicitly for the $2^{1} P^{o}-3^{3} D^{e}$ transition.

In this finite-mass extension of (14), including the electron anomaly, $B_{3 Z}$ requires a factor $\left(\mu / m_{\mathrm{e}}\right)^{3}\left(1+2 a_{\mathrm{e}}+2 y+2 a_{\mathrm{e}} y\right), \Delta_{3}$ requires $\left(\mu / m_{\mathrm{e}}\right)^{3}\left(1+a_{\mathrm{e}}\right) y$ and $B_{3 \mathrm{e}}$ only $\left(\mu / m_{\mathrm{e}}\right)^{3}$ as shown in (14) and (15), while the interaction operators require the mass factors in (15) and (16). If we label the intermediate states with the quantum numbers $m$ and $n$, use nonrelativistic wave functions and reduced energies from (13), and multiply all energies by $\left(\mu / m_{\mathrm{e}}\right)$ to give a.u., the equivalence of the length and velocity formulations in (13) becomes $\Delta \epsilon_{M} M_{\mathrm{LMa}_{\mathrm{e}}}=M_{\mathrm{VMa}_{\mathrm{e}}}+C_{M a_{\mathrm{e}}}$ or

$$
\begin{aligned}
& Z_{r} \frac{\epsilon\left(2^{1} \mathrm{P}^{\mathrm{o}}\right)-\epsilon\left(3^{3} \mathrm{D}\right)}{\left(\mu / m_{\mathrm{e}}\right)}\left[\sum_{m}^{N}\left\langle 3^{3} \mathrm{D}_{2}\left|z_{1}+z_{2}\right| m^{3} \mathrm{P}_{1}^{\mathrm{o}}\right\rangle \frac{\left\langle m^{3} \mathrm{P}_{1}^{\mathrm{o}}\left|B_{3 \mathrm{ZMa}_{\mathrm{e}}}+B_{3 \mathrm{eMa}}\right| 2^{1} \mathrm{P}_{1}^{\mathrm{o}}\right\rangle}{\epsilon\left(2^{1} \mathrm{P}^{\mathrm{o}}\right)-\epsilon\left(m^{3} \mathrm{P}^{\circ}\right)}\right. \\
& +\sum_{m}^{N}\left\langle 3^{3} \mathrm{D}_{1}\left|z_{1}+z_{2}\right| m^{3} \mathrm{P}_{1}^{\mathrm{o}}\right\rangle \frac{\left\langle m^{3} \mathrm{P}_{1}^{\mathrm{o}}\left|B_{3 \mathrm{ZMa}_{\mathrm{e}}}+B_{3 \mathrm{eMa}}\right| 2^{1} \mathrm{P}_{1}^{\mathrm{o}}\right\rangle}{\epsilon\left(2^{1} \mathrm{P}^{\mathrm{o}}\right)-\epsilon\left(m^{3} \mathrm{P}^{\circ}\right)} \\
& \left.+\sum_{n}^{N} \frac{\left\langle 3^{3} \mathrm{D}_{2}\left|B_{3 \mathrm{ZMa}_{\mathrm{e}}}+B_{3 \mathrm{eMa}}\right| n^{1} \mathrm{D}_{2}\right\rangle}{\epsilon\left(3^{3} \mathrm{D}\right)-\epsilon\left(n^{1} \mathrm{D}\right)}\left\langle n^{1} \mathrm{D}_{2}\left|z_{1}+z_{2}\right| 2^{1} \mathrm{P}_{1}^{\mathrm{o}}\right\rangle\right] \\
& =Z_{p}\left[\sum_{m}^{N}\left\langle 3^{3} \mathrm{D}_{2}\left|\frac{\partial}{\partial z_{1}}+\frac{\partial}{\partial z_{2}}\right| m^{3} \mathrm{P}_{1}^{\mathrm{o}}\right\rangle \frac{\left\langle m^{3} \mathrm{P}_{1}^{\mathrm{o}}\left|B_{3 \mathrm{ZMa}_{\mathrm{e}}}+B_{3 \mathrm{eMa}}\right| 2^{1} \mathrm{P}_{1}^{\mathrm{o}}\right\rangle}{\epsilon\left(2^{1} \mathrm{P}^{\mathrm{o}}\right)-\epsilon\left(m^{3} \mathrm{P}^{\mathrm{o}}\right)}\right. \\
& +\sum_{m}^{N}\left\langle 3^{3} \mathrm{D}_{1}\left|\frac{\partial}{\partial z_{1}}+\frac{\partial}{\partial z_{2}}\right| m^{3} \mathrm{P}_{1}^{\mathrm{o}}\right\rangle \frac{\left\langle m^{3} \mathrm{P}_{1}^{\mathrm{o}}\left|B_{3 \mathrm{ZMa}_{\mathrm{e}}}+B_{3 \mathrm{eMa}}\right| 2^{1} \mathrm{P}_{1}^{\mathrm{o}}\right\rangle}{\epsilon\left(2^{1} \mathrm{P}^{\mathrm{o}}\right)-\epsilon\left(m^{3} \mathrm{P}^{\mathrm{o}}\right)}
\end{aligned}
$$

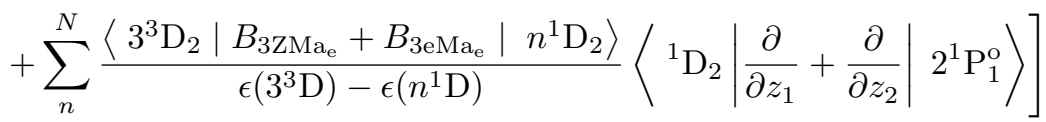

$$
\begin{aligned}
& +C_{M a_{\mathrm{e}}} \text {. }
\end{aligned}
$$

Again for clarity this is the specific example for $2^{1} P_{1}^{o}-3^{3} D_{2}^{e}$ with the $J$-values included to emphasize the need for both angular-momentum and parity equality to obtain non-zero Breit terms, but opposite parity and $\Delta J=0, \pm 1$ for the dipole 
operator (unless both $J$ 's are zero). Thus we must include dipole elements between ${ }^{3} \mathrm{P}_{1}^{\mathrm{o}}$ and both $3{ }^{3} \mathrm{D}_{2}$ and $3^{3} \mathrm{D}_{1}$. Similarly for ${ }^{3} \mathrm{P}_{1,2}^{\mathrm{o}}-{ }^{1} \mathrm{D}_{2}$ there are dipole contributions between ${ }^{3} \mathrm{D}_{2}$ and both ${ }^{3} \mathrm{P}_{1}^{\mathrm{o}}$ and ${ }^{3} \mathrm{P}_{2}^{\mathrm{o}}$.

As described in [13] and [1] we replace each sum over the intermediate states in (18), including the integral over the continuum, by a sum over the asymptotically complete set of $N$ discrete pseudostates obtained by diagonalizing the nonrelativistic Hamiltonian in an orthogonalized basis set of $N$ Hylleraas functions of the appropriate symmetry. Thus we have a set of pseudostates $1 s n p{ }^{3} \mathrm{P}_{1}^{\mathrm{o}}$ interacting with $1 s 2 p{ }^{1} \mathrm{P}_{1}^{\mathrm{o}}$ and $1 s n d{ }^{1} \mathrm{D}_{2}$ interacting with $1 s 3 d{ }^{3} \mathrm{D}_{2}$ in our example of the $1 s 2 p{ }^{1} \mathrm{P}_{1}^{\mathrm{o}}-1 s 3 d{ }^{3} \mathrm{D}_{2}$ transition.

\section{Theory for intermediate coupling}

In Eq. (18) the third and fourth summations use first-order perturbation theory to estimate the effect of the mixing of the ${ }^{1} \mathrm{D}$ and ${ }^{3} \mathrm{D}$ states on the dipole length and velocity matrix elements. When the principal quantum numbers are the same, the energy difference is small allowing that term to dominate all others by more than a factor 100. We can obtain a better value for this term by considering the perturbed two-state system $\left|n^{1} \mathrm{D}\right\rangle$ and $\left|n^{3} \mathrm{D}_{2}\right\rangle$ expanded them in terms of the unperturbed $\left|n^{1} \mathrm{D}_{2}\right\rangle^{0}$ and $\left|n^{3} \mathrm{D}_{2}\right\rangle^{0}$,

$$
\begin{aligned}
& \left|n^{1} \mathrm{D}_{2}\right\rangle=\left|n^{1} \mathrm{D}_{2}\right\rangle^{0} \cos \theta+\left|n^{3} \mathrm{D}_{2}\right\rangle^{0} \sin \theta \\
& \left|n^{3} \mathrm{D}_{2}\right\rangle=-\left|n^{1} \mathrm{D}_{2}\right\rangle^{0} \sin \theta+\left|n^{3} \mathrm{D}_{2}\right\rangle^{0} \cos \theta
\end{aligned}
$$

from which we obtain the matrix equation

$$
\left(\begin{array}{ccc}
\left\langle n{ }^{1} \mathrm{D}_{2}\left|H_{0}\right| n{ }^{1} \mathrm{D}_{2}\right\rangle & \left\langle{ }^{1} \mathrm{D}_{2}\left|B_{3}\right| n^{3} \mathrm{D}_{2}\right\rangle \\
\left\langle n^{3} \mathrm{D}_{2}\left|B_{3}\right| n{ }^{1} \mathrm{D}_{2}\right\rangle & \left\langle n^{3} \mathrm{D}_{2}\left|H_{0}\right| n^{3} \mathrm{D}_{2}\right\rangle
\end{array}\right)=\left(\begin{array}{cc}
E_{11} & B_{12} \\
B_{12} & E_{22}
\end{array}\right)
$$

for the Hamiltonian operator $H=H_{0}+B$. The total perturbation, exact to all orders, is then the sine of the mixing angle $\theta$ required to diagonalize this matrix, where

$$
\frac{\tan 2 \theta}{2}=\frac{B_{12}}{E_{11}-E_{22}} \text {. }
$$

The right-hand side is just the first-order factor in Eq. (18), but in an exact diagonalization both $E_{11}$ and $E_{22}$ include also the diagonal matrix elements $B_{11}$ and $B_{22}$ of the Breit interaction. Drake [8] has calculated $\sin \theta=-0.01560952$ for $3 \mathrm{D}$ and -0.01139597 for $4 \mathrm{D}$ including QED effects as well the nuclear mass and the electron anomaly.

Once we have a corrected length matrix element $\Delta \epsilon_{M} M_{\mathrm{LMa}_{\mathrm{e}}}$ in Eq. (18) we can calculate oscillator strengths $f$ and transition rates $A$

$$
\begin{aligned}
& g_{l} f_{l u}=\frac{2}{3 \Delta \epsilon}\left(\Delta \epsilon_{M} M_{\mathrm{L}}\right)^{2} \\
& g_{u} A_{u l}=\frac{2 \alpha^{4} c}{a_{0}} \Delta \epsilon^{2} g_{l} f_{l u}=3.2130011 \times 10^{10} \Delta \epsilon^{2} g_{l} f_{l u},
\end{aligned}
$$

where $\Delta \epsilon$ is the nonrelativistic energy difference in a.u. and $g_{l}$ and $g_{u}$ are the lower and upper statistical weights respectively. Note that Drake and Morton [5] revised the definition of $f$ in [8] to avoid the use of the $Z_{r}$ and $Z_{p}$ factors in the relation (22) between $f$ and $A$, but $Z_{p}$ still must be applied in calculating $H_{\mathrm{IV}}$ and $Z_{r}$ for $H_{\mathrm{IL}}$ if $Z \neq 2$. 
The relativistic calculations described here used $\alpha=7.2973525376 \times 10^{-3}$ for the fine-structure constant, $a_{\mathrm{e}}=1.159652181 \times 10^{-3}$ for the anomalous magnetic moment of the electron and $y=(\mu / M)=1.37074562 \times 10^{-4}$ for the ratio of the reduced mass of the electron to the alpha particle. All these are within one part in $10^{8}$ or less of the latest recommended values of the fundamental constants [7].

\section{Results and Discussion}

Tables 1 to 4 give the results of our calculations for 12 intersystem $\mathrm{P}-\mathrm{D}$ transitions. The detailed listings give the three components for both length $\Delta \epsilon M_{L}$ and velocity $M_{V}$ matrix elements where the number of figures indicates the level of convergence with increasing size of the basis sets. The correction $C_{\alpha a_{\mathrm{e}}}$ always is better determined. The length-velocity ratio $\left(M_{\mathrm{V}}+C_{\alpha a_{\mathrm{e}}}-\Delta \epsilon M_{\mathrm{L}}\right) / \Delta \epsilon M_{\mathrm{L}}$ provides a guide to the true accuracy of the calculations. Except for a possibly fortuitous $7 \times 10^{-7}$, the ratios range between $2 \times 10^{-5}$ and $4 \times 10^{-4}$. The $f$ - and $A$-values in the tablres are based on $\Delta \epsilon M_{\mathrm{L}}$. Comparison with $f_{2 \times 2}$ indicate that the $\mathrm{P}-\mathrm{D}$ results from the simple $2 \times 2$ diagonalizations are reliable to 2 or 3 significant figures.

As described in Paper III [3], Pachucki [14] identified an additional interaction proportional to $a_{\mathrm{e}}$ due to the magnetic component of the radiation field. The extra contribution to the length matrix element $\Delta \epsilon M_{\mathrm{L}}$ is

$$
P_{M a_{\mathrm{e}}}=-\left(\mu / m_{\mathrm{e}}\right)^{2} a_{\mathrm{e}}\left[B_{\mathrm{I}, 3 Z}-B_{\mathrm{I}, 3 e}\right],
$$

where the factor $\left(\mu / m_{\mathrm{e}}\right)^{2}$ allows for the reduced atomic units appropriate for the finite nuclear mass. This was a small correction for some of the $\mathrm{S}-\mathrm{P}$ transitions in Paper III, but negligible for all the $\mathrm{P}-\mathrm{D}$ cases listed here because both $B_{\mathrm{I}, 3 Z}$ and $B_{\mathrm{I}, 3 e}$ are smaller. As a fraction of $\Delta \epsilon M_{L}$, the two largest $P_{M a_{\mathrm{e}}}$ are $1.84 \times 10^{-7}$ and $1.46 \times 10^{-7}$ for $2{ }^{1} \mathrm{P}_{1}^{\mathrm{o}}-3{ }^{3} \mathrm{D}_{2}$ and $2{ }^{3} \mathrm{P}_{1}^{\mathrm{o}}-3{ }^{1} \mathrm{D}_{2}$.

\section{Future Calculations}

In our studies of ${ }^{1} \mathrm{~S}^{\mathrm{e}}-{ }^{3} \mathrm{P}^{\mathrm{o}}$ and ${ }^{3} \mathrm{~S}^{\mathrm{e}}-{ }^{1} \mathrm{P}^{\mathrm{o}}$ transitions, it was important to include the doubly excited $2 p n p^{3} \mathrm{P}^{\mathrm{e}}$ and $2 p n p{ }^{1} \mathrm{P}^{\mathrm{e}}$ respectively in the sums over intermediate states. Similarly with ${ }^{1} \mathrm{P}^{\mathrm{o}}-{ }^{3} \mathrm{D}^{\mathrm{e}}$ and ${ }^{3} \mathrm{P}^{\mathrm{o}}-{ }^{1} \mathrm{D}^{\mathrm{e}}$ we need to investigate the contributions of the doubly excited $2 p n d{ }^{3} \mathrm{D}^{\mathrm{o}}$ and $2 p n d{ }^{1} \mathrm{D}^{\mathrm{o}}$ states. Also we would like to extend the tables to include higher principal quantum numbers.

\section{Acknowledgments}

Research support by the Natural Sciences and Engineering Research Council of Canada, and by SHARCNET is gratefully acknowledged.

\section{References}

[1] D.C. Morton, P. Moffatt and G.W.F. Drake Can. J. Phys. 89, 129 (2011).

[2] D.C. Morton and G.W.F. Drake Phys. Rev. A 893, 042503 (2011).

[3] D.C. Morton, E.E. Schulhoff and G.W.F. Drake J. Phys. B 48, 235001 (2015).

[4] D.C. Morton and G.W.F. Drake J. Phys. B submitted (2016).

[5] G.W.F. Drake and D.C. Morton Astrophys. J. Suppl. 170, 251 (2007).

[6] G.W.F. Drake, Phys. Rev. A 19, 1387 (1979).

[7] P. J. Mohr, D. B. Newell and B. N. Taylor Rev. Mod. Phys. 88035009 (2016). 
[8] G.W.F. Drake Springer Handbook of Atomic, Molecular and Optical Physics Edited by G.W.F. Drake. (Springer, New York 2006) p. 199.

[9] The wave functions $\psi_{\infty}=Z n(2 S+1) L N . P O W$ and the mass-polarized ones $\psi_{\alpha}=$ $Z n(2 S+1) L N$.POL labelled by $Z, n, S$ and $L$ and the number of terms $N$ are available at http://drake.sharcnet.ca/mediawiki/index.php/Downloadable_Resources.

[10] A.P. Stone Proc. Phys. Soc. (London) 77, 786 (1961); 81, 868 (1963).

[11] G.W.F. Drake and Z.-C. Yan Phys. Rev. A 46, 2378 (1992).

[12] G.W.F. Drake J. Phys. B 9, L169 (1976).

[13] G.W.F. Drake Encyclopedia of Applied Physics vol. 23 (Wiley-VCH 1998) p. 121.

[14] K. Pachucki Phys. Rev. A 67, 012504 (2003); Phys. Rev. A 69, 052502 (2004). 
Table 1. Matrix Elements $M$, oscillator strengths $f$ and rates $A$ for ${ }^{1} \mathrm{P}_{1}^{o}-{ }^{3} \mathrm{D}_{2,1}$ transitions. $M_{\mathrm{L}}$ is the length form, $M_{\mathrm{V}}$ is the velocity form, and $C_{\alpha a_{\mathrm{e}}}$ is the correction to $M_{\mathrm{V}}$. In each case the ${ }^{3} \mathrm{D}$ levels are above the ${ }^{1} \mathrm{P}_{1}^{o}$ level.

\begin{tabular}{|c|c|c|c|}
\hline E1 Transition & $2{ }^{1} \mathrm{P}_{1}^{o}-3{ }^{3} \mathrm{D}_{2,1}$ & $2{ }^{1} \mathrm{P}_{1}^{o}-4{ }^{3} \mathrm{D}_{2,1}$ & $3{ }^{1} \mathrm{P}_{1}^{o}-4{ }^{3} \mathrm{D}_{2,1}$ \\
\hline$\Delta \epsilon$ (a.u.) theory & $6.819112257 \times 10^{-2}$ & $9.253524812 \times 10^{-2}$ & $2.385225514 \times 10^{-2}$ \\
\hline $1 / \lambda\left(\mathrm{cm}^{-1}\right)$ theory & $1.496622149 \times 10^{4}$ & $2.030913947 \times 10^{4}$ & $5.234964903 \times 10^{3}$ \\
\hline$\Delta \epsilon M_{\mathrm{L}}$ from $n^{3} \mathrm{P}_{1}^{o}$ & $-0.111710 \times 10^{-3}$ & $-0.05371 \times 10^{-3}$ & $-0.058671 \times 10^{-3}$ \\
\hline$\Delta \epsilon M_{\mathrm{L}}$ from $n^{3} \mathrm{P}_{1}^{o}$ & $-0.064496 \times 10^{-3}$ & $-0.03101 \times 10^{-3}$ & $-0.033874 \times 10^{-3}$ \\
\hline$\Delta \epsilon M_{\mathrm{L}}$ from $n{ }^{1} \mathrm{D}_{2}$ & $7.286850 \times 10^{-3}$ & $2.54815 \times 10^{-3}$ & $3.008032 \times 10^{-3}$ \\
\hline$\Delta \epsilon M_{\mathrm{L}}$ sum & $7.110644 \times 10^{-3}$ & $2.46342 \times 10^{-3}$ & $2.915488 \times 10^{-3}$ \\
\hline$M_{\mathrm{V}}$ from $n{ }^{3} \mathrm{P}_{1}^{o}$ & $-0.113052 \times 10^{-3}$ & $-0.054598 \times 10^{-3}$ & $-0.058943 \times 10^{-3}$ \\
\hline$M_{\mathrm{V}}$ from $n^{3} \mathrm{P}_{1}^{o}$ & $-0.065271 \times 10^{-3}$ & $-0.031523 \times 10^{-3}$ & $-0.034031 \times 10^{-3}$ \\
\hline$M_{\mathrm{V}}$ from $n^{1} \mathrm{D}_{2}$ & $7.290545 \times 10^{-3}$ & $2.550566 \times 10^{-3}$ & $3.008771 \times 10^{-3}$ \\
\hline$C_{\alpha a_{\mathrm{e}}}$ & $-0.0030389 \times 10^{-3}$ & $-0.0019841 \times 10^{-3}$ & $-0.0006105 \times 10^{-3}$ \\
\hline$M_{\mathrm{V}} \operatorname{sum}+C_{\alpha a_{\mathrm{e}}}$ & $7.109183 \times 10^{-3}$ & $2.462462 \times 10^{-3}$ & $2.915188 \times 10^{-3}$ \\
\hline$M_{\mathrm{V}}+C_{\alpha a_{\mathrm{e}}}-\Delta \epsilon M_{\mathrm{L}}$ & $-1.461 \times 10^{-6}$ & $-9.6 \times 10^{-7}$ & $-3.00 \times 10^{-7}$ \\
\hline$\left(M_{\mathrm{V}}+C_{\alpha a_{\mathrm{e}}}-\Delta \epsilon M_{\mathrm{L}}\right) / \Delta \epsilon M_{\mathrm{L}}$ & $-2.1 \times 10^{-4}$ & $-3.9 \times 10^{-4}$ & $-1.03 \times 10^{-4}$ \\
\hline$f\left({ }^{1} \mathrm{P}_{1}^{o}-{ }^{3} \mathrm{D}_{2}\right)$ & $1.67772 \times 10^{-4}$ & $1.49426 \times 10^{-5}$ & $8.10427 \times 10^{-5}$ \\
\hline$f\left({ }^{1} \mathrm{P}_{1}^{o}-{ }^{3} \mathrm{D}_{1}\right)$ & $1.35557 \times 10^{-8}$ & $2.30943 \times 10^{-6}$ & $1.06901 \times 10^{-8}$ \\
\hline$A\left(\mathrm{~s}^{-1}\right)\left({ }^{3} \mathrm{D}_{2}-{ }^{1} \mathrm{P}_{1}^{o}\right)$ & $1.50397 \times 10^{4}$ & $2.46661 \times 10^{3}$ & $8.88863 \times 10^{2}$ \\
\hline$A\left(\mathrm{~s}^{-1}\right)\left({ }^{3} \mathrm{D}_{1}-{ }^{1} \mathrm{P}_{1}^{o}\right)$ & $2.02529 \times 10^{0}$ & $6.35375 \times 10^{-1}$ & $1.95412 \times 10^{-1}$ \\
\hline$f_{2 x 2}\left({ }^{1} \mathrm{P}_{1}^{o}-{ }^{3} \mathrm{D}_{2}\right)$ & $1.6845 \times 10^{-4}$ & $1.4993 \times 10^{-5}$ & $8.1539 \times 10^{-3}$ \\
\hline
\end{tabular}

Table 2. Matrix Elements $M$, oscillator strengths $f$ and rates $A$ for ${ }^{3} \mathrm{D}_{2,1}-{ }^{1} \mathrm{P}_{1}^{o}$ transitions. $M_{\mathrm{L}}-$ is the length form, $M_{\mathrm{V}}$ is the velocity form, and $C_{\alpha a_{\mathrm{e}}}$ is the correction to $M_{\mathrm{V}}$. In each case the ${ }^{1} \mathrm{P}_{1}^{o}$ level is above the ${ }^{3} \mathrm{D}$ levels.

\begin{tabular}{lccc}
\hline E1 Transition & $3{ }^{3} \mathrm{D}_{2,1}-3{ }^{1} \mathrm{P}_{1}^{o}$ & $3{ }^{3} \mathrm{D}_{2,1}-4{ }^{1} \mathrm{P}_{1}^{o}$ & $4{ }^{3} \mathrm{D}_{2,1}-4{ }^{1} \mathrm{P}_{1}^{o}$ \\
\hline$\Delta \epsilon($ a.u. $)$ theory & $4.918704127 \times 10^{-4}$ & $2.456414571 \times 10^{-2}$ & $2.200201604 \times 10^{-4}$ \\
$1 / \lambda\left(\mathrm{cm}^{-1}\right)$ theory & $1.079530775 \times 10^{2}$ & $5.391206825 \times 10^{3}$ & $4.828884361 \times 10^{1}$ \\
& & & \\
& & & \\
$\Delta \epsilon M_{\mathrm{L}}$ from $n{ }^{3} \mathrm{P}_{1}^{o}$ & $-0.015543180 \times 10^{-4}$ & $-0.089690 \times 10^{-4}$ & $-0.1405942 \times 10^{-5}$ \\
$\Delta \epsilon M_{\mathrm{L}}$ from $n{ }^{3} \mathrm{P}_{1}^{o}$ & $-0.008973859 \times 10^{-4}$ & $-0.051783 \times 10^{-4}$ & $-0.0811721 \times 10^{-5}$ \\
$\Delta \epsilon M_{\mathrm{L}}$ from $n{ }^{1} \mathrm{D}_{2}$ & $1.086645859 \times 10^{-4}$ & $6.397072 \times 10^{-4}$ & $7.3386655 \times 10^{-5}$ \\
$\Delta \epsilon M_{\mathrm{L}}$ sum & $1.062128820 \times 10^{-4}$ & $6.255599 \times 10^{-4}$ & $7.1168991 \times 10^{-5}$ \\
$M_{\mathrm{V}}$ from $n{ }^{3} \mathrm{P}_{1}^{o}$ & $-0.0157278 \times 10^{-4}$ & $-0.089158 \times 10^{-4}$ & $-0.14154 \times 10^{-5}$ \\
$M_{\mathrm{V}}$ from $n{ }^{3} \mathrm{P}_{1}^{o}$ & $-0.0090804 \times 10^{-4}$ & $-0.051475 \times 10^{-4}$ & $-0.08172 \times 10^{-5}$ \\
$M_{\mathrm{V}}$ from $n{ }^{1} \mathrm{D}_{2}$ & $1.0869910 \times 10^{-4}$ & $6.395753 \times 10^{-4}$ & $7.34057 \times 10^{-5}$ \\
$C_{\alpha a_{\mathrm{e}}}$ & $-0.000205542 \times 10^{-4}$ & $0.0010899 \times 10^{-4}$ & $-0.0010977 \times 10^{-4}$ \\
$M_{\mathrm{V}}$ sum $+C_{\alpha a_{\mathrm{e}}}$ & $1.0619772 \times 10^{-4}$ & $6.256210 \times 10^{-4}$ & $7.11622 \times 10^{-4}$ \\
$M_{\mathrm{V}}+C_{\alpha a_{\mathrm{e}}}-\Delta \epsilon M_{\mathrm{L}}$ & $-1.516 \times 10^{-8}$ & $6.11 \times 10^{-8}$ & $6.80 \times 10^{-9}$ \\
$\left(M_{\mathrm{V}}+C_{\alpha a_{\mathrm{e}}}-\Delta \epsilon M_{\mathrm{L}}\right) / \Delta \epsilon M_{\mathrm{L}}$ & $-1.4 \times 10^{-4}$ & $9.8 \times 10^{-5}$ & $-9.6 \times 10^{-5}$ \\
& & & \\
$f\left({ }^{3} \mathrm{D}_{2}-{ }^{1} \mathrm{P}_{1}^{o}\right)$ & $3.10993 \times 10^{-6}$ & $2.15941 \times 10^{-6}$ & $3.13985 \times 10^{-6}$ \\
$f\left({ }^{3} \mathrm{D}_{1}-{ }^{1} \mathrm{P}_{1}^{o}\right)$ & $3.63827 \times 10^{-10}$ & $2.42580 \times 10^{-10}$ & $6.65486 \times 10^{-10}$ \\
$A\left(\mathrm{~s}^{-1}\right)\left({ }^{1} \mathrm{P}_{0}^{o}-{ }^{3} \mathrm{D}_{2}\right)$ & $4.02913 \times 10^{-2}$ & $6.97747 \times 10^{1}$ & $8.13941 \times 10^{-3}$ \\
$A\left(\mathrm{~s}^{-1}\right)\left({ }^{1} \mathrm{P}_{1}^{o}-{ }^{3} \mathrm{D}_{1}\right)$ & $2.82818 \times 10^{-6}$ & $4.70294 \times 10^{-3}$ & $1.03508 \times 10^{-6}$ \\
$f_{2 x 2}\left({ }^{3} \mathrm{D}_{2}-{ }^{1} \mathrm{P}_{1}^{o}\right)$ & & & \\
$f_{2 x 2}\left({ }^{3} \mathrm{D}_{1}-{ }^{1} \mathrm{P}_{1}^{o}\right)$ & $3.1008 \times 10^{-6}$ & $2.1408 \times 10^{-6}$ & $3.1318 \times 10^{-6}$ \\
& $3.6735 \times 10^{-10}$ &. & $6.6338 \times 10^{-10}$ \\
\hline
\end{tabular}


Table 3. Matrix Elements $M$, oscillator strengths $f$ and rates $A$ for ${ }^{3} \mathrm{P}_{1,2}^{o}-{ }^{1} \mathrm{D}_{2}$ transitions. $M_{\mathrm{L}}$ is the length form, $M_{\mathrm{V}}$ is the velocity form, and $C_{\alpha a_{\mathrm{e}}}$ is the correction to $M_{\mathrm{V}}$. In each case the ${ }^{3} \mathrm{D}_{2}$ level is above the ${ }^{1} \mathrm{P}^{o}$ levels.

\begin{tabular}{|c|c|c|c|}
\hline E1 Transition & $2{ }^{3} \mathrm{P}_{1,2}^{o}-3{ }^{1} \mathrm{D}_{2}$ & $2^{3} \mathrm{P}_{1,2}^{o}-4{ }^{1} \mathrm{D}_{2}$ & $3{ }^{3} \mathrm{P}_{1,2}^{o}-3{ }^{1} \mathrm{D}_{2}$ \\
\hline$\Delta \epsilon$ (a.u.) theory & $7.754164731 \times 10^{-2}$ & $1.018 .792144 \times 10^{-1}$ & $2.462497812 \times 10^{-3}$ \\
\hline $1 / \lambda\left(\mathrm{cm}^{-1}\right)$ theory & $1.701842446 \times 10^{4}$ & $2.235990303 \times 10^{4}$ & $5.404557996 \times 10^{2}$ \\
\hline$\Delta \epsilon M_{\mathrm{L}}$ from $n{ }^{1} \mathrm{P}_{1}^{o}$ & $-0.130571 \times 10^{-3}$ & $-0.06699 \times 10^{-3}$ & $-0.0912691 \times 10^{-4}$ \\
\hline$\Delta \epsilon M_{\mathrm{L}}$ from $n^{3} \mathrm{D}_{2}$ & $6.234804 \times 10^{-3}$ & $2.34257 \times 10^{-3}$ & $4.7828962 \times 10^{-4}$ \\
\hline$\Delta \epsilon M_{\mathrm{L}}$ from $n^{3} \mathrm{D}_{2}$ & $3.599666 \times 10^{-3}$ & $1.35249 \times 10^{-3}$ & $2.7614064 \times 10^{-4}$ \\
\hline$\Delta \epsilon M_{\mathrm{L}}$ sum & $9.703899 \times 10^{-3}$ & $3.62807 \times 10^{-3}$ & $7.4530334 \times 10^{-4}$ \\
\hline$M_{\mathrm{V}}$ from $n{ }^{1} \mathrm{P}_{1}^{o}$ & $-0.1290538 \times 10^{-3}$ & $-0.065966 \times 10^{-3}$ & $-0.090265 \times 10^{-4}$ \\
\hline$M_{\mathrm{V}}$ from $n^{3} \mathrm{D}_{2}$ & $6.2316143 \times 10^{-3}$ & $2.340443 \times 10^{-3}$ & $4.780977 \times 10^{-4}$ \\
\hline$M_{\mathrm{V}}$ from $n^{3} \mathrm{D}_{2}$ & $3.5996660 \times 10^{-3}$ & $1.351256 \times 10^{-3}$ & $2.760299 \times 10^{-4}$ \\
\hline$C_{\alpha a_{\mathrm{e}}}$ & $0.0030462 \times 10^{-3}$ & $0.0020321 \times 10^{-3}$ & $0.0018071 \times 10^{-4}$ \\
\hline$M_{\mathrm{V}}$ sum $+C_{\alpha a_{\mathrm{e}}}$ & $9.7034308 \times 10^{-3}$ & $3.627765 \times 10^{-3}$ & $7.452818 \times 10^{-4}$ \\
\hline$M_{\mathrm{V}}+C_{\alpha a_{\mathrm{e}}}-\Delta \epsilon M_{\mathrm{L}}$ & $-4.684 \times 10^{-7}$ & $-3.069 \times 10^{-7}$ & $-2.153 \times 10^{-8}$ \\
\hline$\left(M_{\mathrm{V}}+C_{\alpha a_{\mathrm{e}}}-\Delta \epsilon M_{\mathrm{L}}\right) / \Delta \epsilon M_{\mathrm{L}}$ & $-4.8 \times 10^{-5}$ & $-8.5 \times 10^{-5}$ & $-2.9 \times 10^{-5}$ \\
\hline$f\left({ }^{3} \mathrm{P}_{1}^{o}-{ }^{1} \mathrm{D}_{2}\right)$ & $1.06786 \times 10^{-4}$ & $1.12951 \times 10^{-5}$ & $1.98636 \times 10^{-5}$ \\
\hline$f\left({ }^{3} \mathrm{P}_{2}^{o}-{ }^{1} \mathrm{D}_{2}\right)$ & $2.22807 \times 10^{-5}$ & $2.39397 \times 10^{-6}$ & $4.12880 \times 10^{-6}$ \\
\hline$A\left(\mathrm{~s}^{-1}\right)\left({ }^{1} \mathrm{D}_{2}-{ }^{3} \mathrm{P}_{1}^{o}\right)$ & $1.23779 \times 10^{4}$ & $2.26007 \times 10^{3}$ & $2.32205 \times 10^{0}$ \\
\hline$A\left(\mathrm{~s}^{-1}\right)\left({ }^{1} \mathrm{D}_{2}-{ }^{3} \mathrm{P}_{2}^{o}\right)$ & $4.30436 \times 10^{3}$ & $7.98363 \times 10^{2}$ & $8.04426 \times 10^{-1}$ \\
\hline$f_{2 x 2}\left({ }^{3} \mathrm{P}_{1}^{o}-{ }^{1} \mathrm{D}_{2}\right)$ & $1.0632 \times 10^{-4}$ & $1.1276 \times 10^{-5}$ & $1.9859 \times 10^{-5}$ \\
\hline$f_{2 x 2}\left({ }^{3} \mathrm{P}_{2}^{o}-{ }^{1} \mathrm{D}_{2}\right)$ & $2.2307 \times 10^{-5}$ & $2.3933 \times 10^{-6}$ & $4.1232 \times 10^{-6}$ \\
\hline
\end{tabular}


Table 4. Matrix Elements $M$, oscillator strengths $f$ and rates $A$ for ${ }^{3} \mathrm{P}_{1,2}^{o}-{ }^{1} \mathrm{D}_{2}$ transitions. $M_{\mathrm{L}}$ is the length form, $M_{\mathrm{V}}$ is the velocity form, and $C_{\alpha a_{\mathrm{e}}}$ is the correction to $M_{\mathrm{V}}$. The $4{ }^{1} \mathrm{D}_{2}$ level is above the 3 and $4{ }^{3} \mathrm{P}_{1,2}^{o}$ levels, but $4{ }^{3} \mathrm{P}_{1}^{o}$ is above $3{ }^{1} \mathrm{D}_{2}$.

\begin{tabular}{|c|c|c|c|}
\hline E1 Transition & $3{ }^{3} \mathrm{P}_{1,2}^{o}-4{ }^{1} \mathrm{D}_{2}$ & $3{ }^{1} \mathrm{D}_{2}-4{ }^{3} \mathrm{P}_{1,2}^{o}$ & $4{ }^{3} \mathrm{P}_{1,2}^{o}-4{ }^{1} \mathrm{D}_{2}$ \\
\hline$\Delta \epsilon$ (a.u.) theory & $2.680006492 \times 10^{-2}$ & $2.329218431 \times 10^{-2}$ & $1.045382794 \times 10^{-3}$ \\
\hline $1 / \lambda\left(\mathrm{cm}^{-1}\right)$ theory & $5.881934369 \times 10^{3}$ & $5.112043566 \times 10^{3}$ & $2.294350033 \times 10^{2}$ \\
\hline $\begin{array}{l}\Delta \epsilon M_{\mathrm{L}} \text { from } n{ }^{1} \mathrm{P}_{1}^{o} \\
\Delta \epsilon M_{\mathrm{L}} \text { from } n^{3} \mathrm{D}_{2} \\
\Delta \epsilon M_{\mathrm{L}} \text { from } n^{3} \mathrm{D}_{2} \\
\Delta \epsilon M_{\mathrm{L}} \text { sum } \\
M_{\mathrm{V}} \text { from } n{ }^{1} \mathrm{P}_{1}^{o} \\
M_{\mathrm{V}} \text { from } n^{3} \mathrm{D}_{2} \\
M_{\mathrm{V}} \text { from } n{ }^{3} \mathrm{D}_{2} \\
C_{\alpha a_{\mathrm{e}}} \\
M_{\mathrm{V}} \text { sum }+C_{\alpha a_{\mathrm{e}}} \\
M_{\mathrm{V}}+C_{\alpha a_{\mathrm{e}}}-\Delta \epsilon M_{\mathrm{L}} \\
\left(M_{\mathrm{V}}+C_{\alpha a_{\mathrm{e}}}-\Delta \epsilon M_{\mathrm{L}}\right) / \Delta \epsilon M_{\mathrm{L}}\end{array}$ & $\begin{array}{l}-0.062137 \times 10^{-3} \\
2.365030 \times 10^{-3} \\
1.365451 \times 10^{-3} \\
3.668344 \times 10^{-3} \\
-0.061908 \times 10^{-3} \\
2.364543 \times 10^{-3} \\
1.365169 \times 10^{-3} \\
0.00047265 \times 10^{-3} \\
3.668276 \times 10^{-3} \\
-6.8 \times 10^{-8} \\
-1.9 \times 10^{-5}\end{array}$ & $\begin{array}{r}-0.0156942 \times 10^{-3} \\
0.8431701 \times 10^{-3} \\
0.4868045 \times 10^{-3} \\
1.3142804 \times 10^{-3} \\
-0.0157009 \times 10^{-3} \\
0.8431908 \times 10^{-3} \\
0.4868164 \times 10^{-3} \\
-0.0000269 \times 10^{-3} \\
1.3142795 \times 10^{-3} \\
-1.0 \times 10^{-9} \\
-7 . \times 10^{-7}\end{array}$ & $\begin{array}{r}-0.0779247 \times 10^{-4} \\
3.0494245 \times 10^{-4} \\
1.7605860 \times 10^{-4} \\
4.7320858 \times 10^{-4} \\
-0.077432 \times 10^{-4} \\
3.048499 \times 10^{-4} \\
1.760052 \times 10^{-4} \\
0.0008799 \times 10^{-4} \\
4.731998 \times 10^{-4} \\
-8.8 \times 10^{-9} \\
-1.8 \times 10^{-5}\end{array}$ \\
\hline $\begin{array}{c}f\left({ }^{3} \mathrm{P}_{1}^{o}-{ }^{1} \mathrm{D}_{2}\right) \\
f\left({ }^{1} \mathrm{D}_{2}-{ }^{3} \mathrm{P}^{o}\right)\end{array}$ & $4.39743 \times 10^{-5}$ & $91958 \times 10^{-6}$ & $1.87700 \times 10^{-5}$ \\
\hline $\begin{array}{c}f\left({ }^{3} \mathrm{P}_{2}^{o}-{ }^{1} \mathrm{D}_{2}\right) \\
f\left({ }^{1} \mathrm{D}_{2}-{ }^{3} \mathrm{P}_{2}^{o}\right)\end{array}$ & $9.27588 \times 10^{-6}$ & $1.35656 \times 10^{-6}$ & $3.95347 \times 10^{-6}$ \\
\hline $\begin{array}{l}A\left(\mathrm{~s}^{-1}\right)\left({ }^{1} \mathrm{D}_{2}-3 \mathrm{P}_{1}^{o}\right) \\
A\left(\mathrm{~s}^{-1}\right)\left({ }^{3} \mathrm{P}_{1}^{o}-{ }^{1} \mathrm{D}_{2}\right)\end{array}$ & $6.08882 \times 10^{2}$ & $1.13873 \times 10^{2}$ & $3.95436 \times 10^{-1}$ \\
\hline $\begin{array}{l}A\left(\mathrm{~s}^{-1}\right)\left({ }^{1} \mathrm{D}_{2}-3{ }^{-} \mathrm{P}_{2}^{o}\right) \\
\quad A\left(\mathrm{~s}^{-1}\right)\left({ }^{3} \mathrm{P}_{2}^{o}-{ }^{1} \mathrm{D}_{2}\right)\end{array}$ & $\begin{array}{l}2.14061 \times 10^{2} \\
.\end{array}$ & $2.36466 \times 10^{2}$ & $1.38816 \times 10^{-1}$ \\
\hline $\begin{array}{c}f_{2 x 2}\left({ }^{3} \mathrm{P}_{1}^{o}-{ }^{1} \mathrm{D}_{2}\right) \\
f_{2 x 2}\left({ }^{1} \mathrm{D}_{2}-{ }^{3} \mathrm{P}_{1}^{\mathrm{o}}\right)\end{array}$ & $4.3605 \times 10^{-5}$ & $3.9580 \times 10^{-6}$ & $1.8765 \times 10^{-5}$ \\
\hline $\begin{array}{c}f_{2 x 2}\left({ }^{3} \mathrm{P}_{2}^{o}-{ }^{1} \mathrm{D}_{2}\right) \\
f_{2 x 2}\left({ }^{1} \mathrm{D}_{2}-{ }^{3} \mathrm{P}_{2}^{o}\right)\end{array}$ & $9.3067 \times 10^{-6}$ & $1.3504 \times 10^{-6}$ & $3.9485 \times 10^{-6}$ \\
\hline
\end{tabular}

